\title{
Perfil de usuários em um serviço de pronto atendimento
}

\section{Profile of users in an emergency care}

Mariana Figueiredo Souza Gomide"; Ione Carvalho Pinto²; Daniela Martorano Pires Gomide ${ }^{3}$; Fabiana Costa Machado Zacharias ${ }^{4}$

\begin{abstract}
RESUMO
O objetivo deste estudo foi identificar o perfil de usuários atendidos no pronto atendimento (PA) de um distrito do município de Ribeirão Preto - SP. Estudo quantitativo sobre usuários que procuraram o PA entre janeiro a junho de 2009. Foram caracterizados quanto ao sexo, faixa etária, horário, dia da semana e mês de maior procura, justificativa da procura, conduta e encaminhamentos realizados. Dos 45.771 atendimentos realizados, foi selecionada amostra com 330 fichas de atendimento pela técnica de amostragem aleatória. Desta amostra, $57 \%$ dos usuários eram do sexo feminino e $63,3 \%$ são usuários entre 20 a 50 anos. Os meses de maior procura foram março, abril e maio e 70,7\% dos atendimentos ocorreram durante o dia. Em $20 \%$ das justificativas tiveram o relato de dor. Em $77,2 \%$ das condutas estão a terapia medicamentosa e $26,3 \%$ a solicitação de exames. Entre as diversas justificativas para a procura pelo PA estão as potencialidades deste serviço na forma de alta densidade tecnológica.
\end{abstract}

Palavras-chave: Atenção Primária à Saúde. Acesso aos Serviços de Saúde. Serviços Médicos de Emergência.

\section{Introdução}

O cuidado em saúde no Sistema Único de Saúde (SUS) foi ordenado em níveis de atenção, assim, temos a assistência básica, a média e a de alta complexidade, visando à melhor programação e planejamento das ações e serviços do sistema de saúde.

Nesse sentido, as Unidades Básicas de Saúde (UBS) compõem a estrutura física básica de atendimento aos usuários do SUS. Quando funcionam adequadamente, a população consegue resolver a maio- ria dos seus problemas de saúde e, por conseguinte, podem reduzir as filas nos níveis de média e alta complexidade que correspondem às Unidades Básicas Distritais de Saúde (UBDS) e à assistência hospitalar de especialidades respectivamente. Além disso, também reduzem o consumo abusivo de medicamentos e o uso indiscriminado de equipamentos de alta tecnologia. ${ }^{1}$

Assim, os problemas de saúde mais comuns passam a ser resolvido nas UBS, deixando os ambulatórios de especialidades e hospitais cumprirem seus
1. Enfermeira, mestre em Ciências pelo Programa de Enfermagem em Saúde Pública da Escola de Enfermagem de Ribeirão Preto da Universidade de São Paulo (EERP-USP).

2. Docente. Departamento de Enfermagem Materno Infantil e Saúde Pública da EERP-USP.

3. Estatística, graduada pela Universidade Estadual de Campinas.

4. Pós Graduanda (mestrado) do Programa de Pós Graduação de Enfermagem em Saúde Pública da EERP-USP
Correspondência: mari_figueiredo@ig.com.br

Artigo recebido em 01/07/2011 Aprovado para publicação em 26/01/2012 
verdadeiros papéis, resultando maior satisfação dos usuários e utilização mais racional dos recursos existentes. ${ }^{1}$

As UBS têm como principal fundamento ser a porta de entrada preferencial ao sistema de saúde. Além de integrar as ações programáticas e de demanda espontânea; articular ações de promoção à saúde, prevenção de agravos, vigilância à saúde, tratamento e reabilitação, trabalho em equipe, desenvolver relações de vínculo e responsabilização entre as equipes e a população adstrita, garantindo a continuidade das ações de saúde e a longitudinalidade do cuidado; estimulando a participação popular e o controle social. ${ }^{1}$

Nas UBDS têm-se interligadas as unidades de pronto-atendimento (PA), que são estruturas de complexidade intermediária entre as UBS e as portas de urgência hospitalares, e que em conjunto com aquelas compõem uma rede organizada de atenção às urgências.

O PA se constitui em um serviço organizado para funcionar 24horas/dia ininterruptamente, para atender a qualquer pessoa que procure, sem agendamento prévio ou limite de consultas, com caráter de urgência ou emergência. ${ }^{2}$

Consideraram-se os conceitos de urgência e emergência sendo a primeira a ocorrência imprevista de agravo à saúde com ou sem risco potencial de vida, e a segunda, como a constatação médica de condições de agravo à saúde que impliquem em risco iminente de vida. ${ }^{3}$

Essa estratégia de atendimento está diretamente relacionada ao trabalho do Serviço Móvel de Urgência - SAMU que organiza o fluxo de atendimento e encaminha o paciente ao serviço de saúde adequado a cada situação.

Em razão do crescente número de atendimentos nessas unidades, neste estudo, têm-se como pressuposto que, mesmo sendo serviço de urgência, o PA atende a prontidões e também atende aos usuários considerados não urgentes, resultando no aumento da demanda para casos que não seguem os critérios de urgência e emergência.

O objetivo deste estudo foi identificar o perfil de usuários atendidos no PA do distrito oeste do município de Ribeirão Preto - SP segundo o sexo, faixa etária, horário, dia da semana e mês de atendimento, justificativa para procura pelo PA, conduta e encaminhamentos realizados.

\section{Materiais e métodos}

Este estudo tem abordagem quantitativa sobre os usuários que procuraram o PA no período de janeiro a junho de 2009.

O local de estudo foi o Pronto-Atendimento do Centro de Saúde Escola da Faculdade de Medicina de Ribeirão Preto da Universidade de São Paulo - CSE/ FMRP-USP localizado no Distrito Oeste do município de Ribeirão Preto.

Foi realizada uma busca ao banco de dados Hygia da Secretaria Municipal de Saúde (SMS) de Ribeirão Preto-SP e foi encontrado que, no período estudado, ou seja, entre $1^{\circ}$ de janeiro de 2009 e 30 de junho de 2009, foram prestados 45.771 atendimentos para 25.110 usuários.

Nessa busca, também foi obtido dados relativos de todos os usuários que foram atendidos nesse período segundo sexo, faixa etária, horário, dia da semana e mês do atendimento no PA.

Porém, para se obter os dados quanto à justificativa da procura, condutas e encaminhamentos realizados por parte dos profissionais que prestaram o atendimento, foi necessário recorrer às fichas de registro de atendimento.

Nestas fichas, além de haver a identificação dos usuários que procuraram o PA segundo as características acima relatadas, elas também contêm os registros de justificativa de procura pelo PA que são realizados pelos profissionais de enfermagem durante o acolhimento, e as condutas e os encaminhamentos realizados, preenchidos pelos médicos que prestaram $\mathrm{o}$ atendimento.

Para isso, foi necessária a seleção de uma amostra obtida a partir dos 45.771 atendimentos, com a finalidade de buscar informações sobre quais foram as principais justificativas para os usuários procurarem atendimento no PA e quais foram as condutas e os encaminhamentos realizados por parte dos profissionais que os atenderam.

Foi selecionada uma amostra das fichas de atendimento, a partir da técnica de Amostragem Aleatória Sistemática ${ }^{4,5}$ que garante que todos os atendimentos tiveram a mesma probabilidade de estar nesta amostra, pois seria inviável levantar todas as fichas do período.

Para o cálculo do tamanho da amostra, é preciso conhecer alguns parâmetros. 


$$
\mathrm{n}=\frac{N \cdot \hat{p} \cdot \hat{q} \cdot\left(\mathrm{Z}_{\alpha / 2}\right)^{2}}{\hat{p} \cdot \hat{q} \cdot\left(\mathrm{Z}_{\alpha / 2}\right)^{2}+(\mathrm{N}-1) \cdot E^{2}}
$$

Nessa fórmula, $\mathrm{n}$ representa o tamanho da amostra, p corresponde à proporção de usuários que não precisariam estar sendo atendidos no PA, q = 1-p, ou seja, é o percentual das consultas necessárias, E representa o erro amostral tolerado e $\mathrm{Z}$ corresponde ao grau de confiança desejado.

Neste estudo, não se conhece a proporção de usuários que procuram indevidamente pelo atendimento no PA, nestes casos é possível assumir uma proporção de 0,5 , garantindo um tamanho de amostra adequado.

O erro amostral aceito foi de $5 \%$ e o grau de confiança foi de $90 \%$.

Dessa forma, tem-se que a amostra ideal deveria ter 270 atendimentos. Como houve problemas para a localização das fichas selecionadas e problemas quanto ao seu preenchimento, foi extrapolada a quantidade e validada 330 fichas de atendimento, quantidade que garante os parâmetros usados, pois está acima da quantidade ideal.

\section{Análise dos dados}

Para a apresentação dos resultados encontrados, a partir da coleta de dados do banco Hygia e da amostra utilizada, foi feito a apresentação da caracte- rização dos usuários segundo sexo, faixa etária e período do atendimento de forma descritiva e também com dados distribuídos em tabelas e gráficos.

Foram analisados os dados relativos às justificativas para procura pelo PA a partir do que era registrado nas fichas de atendimento pelos profissionais de enfermagem, no momento do acolhimento que antecede os atendimentos propriamente ditos, e foram analisados os dados relativos às condutas realizadas no PA a partir do que era registrado no campo evolução médica nas fichas de atendimento pelos profissionais médicos após o atendimento.

Obteve-se autorização ao Comitê de Ética em Pesquisa do CSE/FMRP-USP sob $n^{\circ}$ de protocolo 049/ 2009, seguindo assim, as normatizações da Comissão Nacional de Ética em Pesquisa - CONEP, presentes na Resolução do CNS 196/96 e capítulo da Resolução 251/97.

\section{Resultados}

São apresentados inicialmente dados relativos ao sexo, faixa etária, horário, dia da semana e mês de atendimento da amostra utilizada, ou seja, trata-se de 330 fichas de atendimento de pacientes atendidos no PA entre $1^{\circ}$ de janeiro de 2009 e 30 de junho de 2009.

Desta amostra, tem-se que $57 \%$ dos usuários eram do sexo feminino e $43 \%$ do sexo masculino.

O gráfico 1 mostra a distribuição dos usuários, a partir da amostra utilizada, que procuraram o PA conforme a faixa etária.

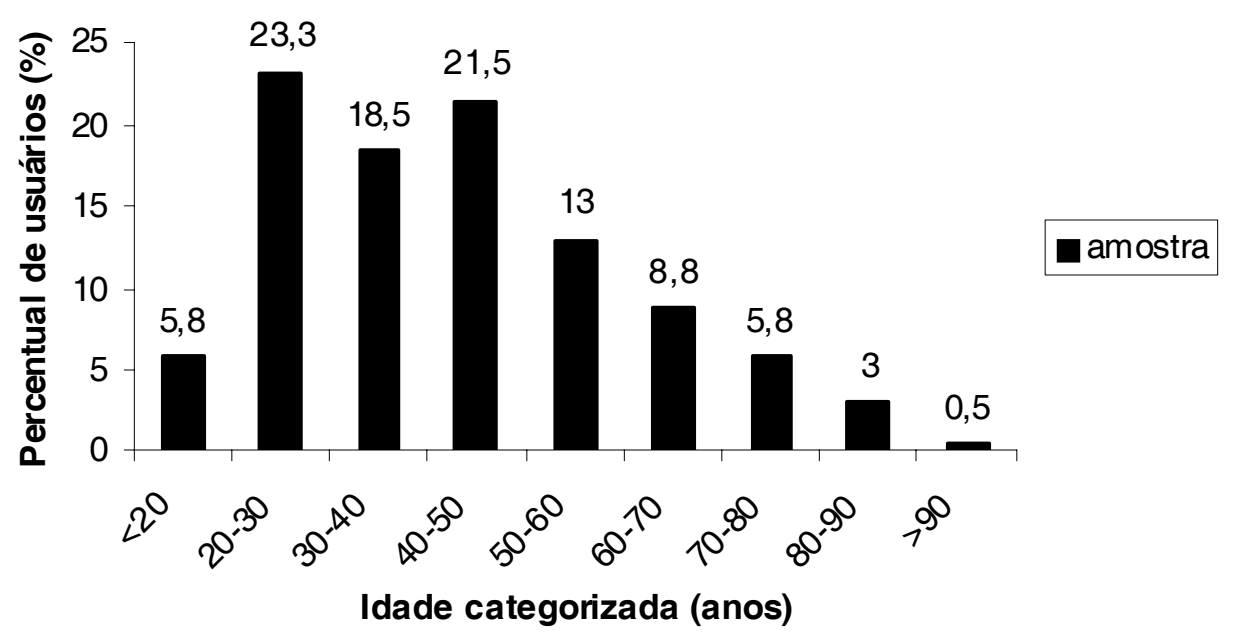

Gráfico 1: Distribuição dos usuários atendidos no PA do CSE/FMRP-USP, no período de 1º de janeiro a 30 de junho de 2009 , quanto à faixa etária. 
Na distribuição entre os meses do período estudado da amostra utilizada encontrou-se que 16,5\% dos usuários procuraram atendimento em janeiro, $16 \%$ em fevereiro, $17,6 \%$ em março, $17,4 \%$ em abril, $17,7 \%$ em maio, e $14,5 \%$ em junho.

Quanto ao período de atendimento que os usuários procuraram o PA, obteve-se como resultado que $6,7 \%$ procuraram atendimento de madrugada, $39 \%$ procuraram no período matutino, $31,7 \%$ no período vespertino e $22,5 \%$ no periodo noturno.
O gráfico 3 traz o cruzamento de dados entre a proporção de usuários que procuraram atendimento no PA nos diferentes períodos do dia conforme a faixa etária.

Na tabela 1, pôde-se identificar inúmeras justificativas para os usuários terem procurado o PA. Este agrupamento considerou o que estava registrado nas fichas de atendimento pelo profissional de enfermagem, no acolhimento, sobre o que motivou a ida do usuário ao serviço.

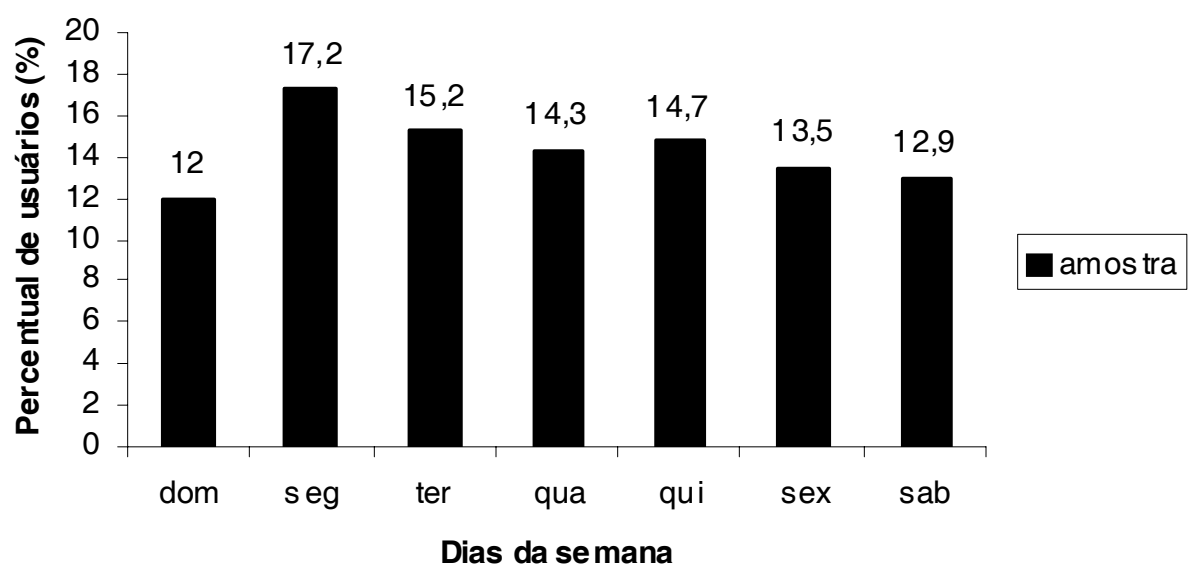

Gráfico 2: Distribuição dos usuários atendidos no PA do CSE/FMRP-USP, no período de 1ำ de janeiro a 30 de junho de 2009 , quanto à frequência de atendimento entre os dias da semana.

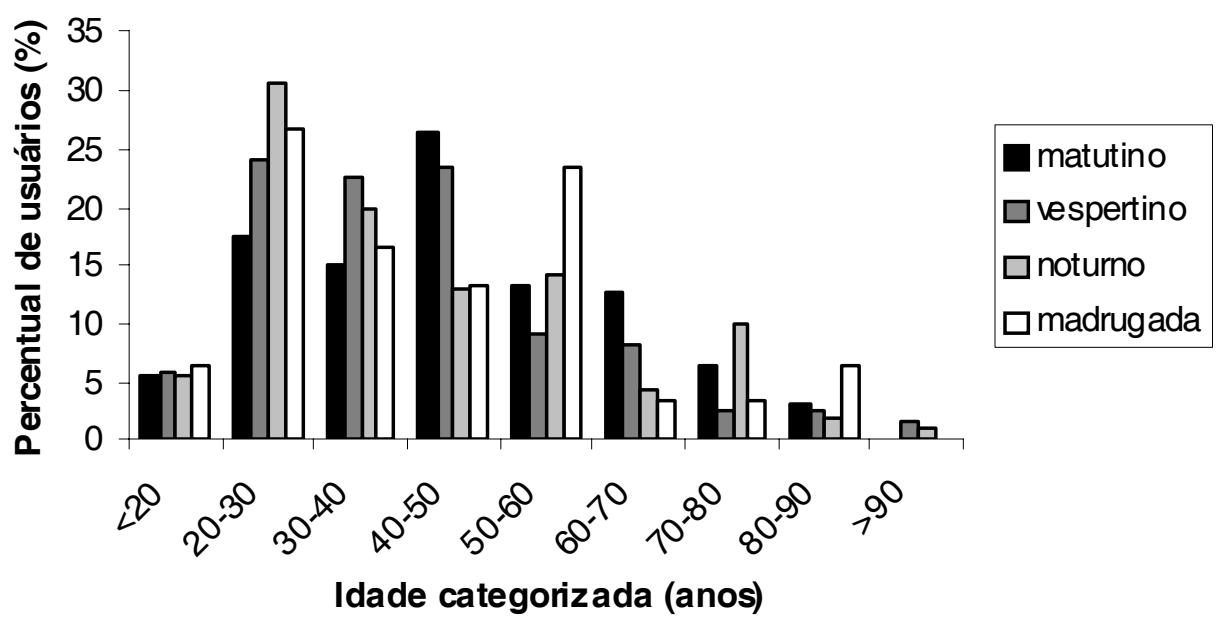

Gráfico 3: Distribuição de usuários atendidos no PA do CSE/FMRP-USP, no período de 1o de janeiro a 30 de junho de 2009 , quanto à frequência de atendimento entre o período do dia e faixa etária. 
Logo, sintomas registrados nas fichas como gripe, tosse e dor de garganta, classificaram-se como problemas respiratórios. Sintomas como dor epigástrica, náuseas e diarréia, classificaram-se como problemas gastrointestinais, e assim por diante foi realizado o agrupamento de justificativas para a procura pelo PA.

\section{Tabela 1}

Distribuição dos usuários selecionados na amostra quanto à justificativa para a procura pelo PA do CSE/ FMRP-USP, no período de $1^{\circ}$ de janeiro a 30 de junho de 2009

\begin{tabular}{lcc}
\hline Justificativa da procura pelo PA & N & \% \\
\hline Dor & 68 & 20 \\
Trauma/ lesão & 52 & 15,7 \\
Problema respiratório & 51 & 15,4 \\
Problema gastrointestinal & 37 & 11,2 \\
Mal estar inespecífico & 22 & 6,6 \\
Problema renal & 18 & 5,4 \\
Problemas Cardiovasculares & 16 & 4 \\
Problema ginecológico & 14 & 4,2 \\
Checar exames & 14 & 4,2 \\
Sintomatologia de dengue & 8 & 2,4 \\
Crise alérgica & 7 & 2,1 \\
Problema oftalmológico & 6 & 1,8 \\
Administração de medicamentos & 2 & 0,6 \\
DM descompensada & 2 & 0,6 \\
Outras causas & 13 & 3,6 \\
\hline Total & $\mathbf{3 3 0}$ & $\mathbf{1 0 0 , 0}$ \\
\hline
\end{tabular}

Entre os 20\% relatos de dor, 32,3\% dos usuários relataram dor nos membros, $17,6 \%$ relataram dor em mais de uma região do corpo, $8,8 \%$, cefaléias, $30,8 \%$ relataram lombalgias e $10,2 \%$, dores torácicas.

No item "outras causas", entre os resultados foram encontrados que pelo menos $2 \%$ dos usuários justificaram a procura pelo PA por encaminhamentos das UBSs, onde se encontrava registrado por algum profissional da USF: "médico de férias". Este também foi resultado de outro estudo. ${ }^{6}$

A tabela 2 traz dados relativos às fichas de atendimento quanto às condutas registradas pelos profissionais médicos.

\section{Tabela 2}

Distribuição dos usuários selecionados na amostra quanto à conduta realizada pelo médico nos atendimentos realizados no PA do CSE de Ribeirão Preto, no período de $1^{\circ}$ de janeiro a 30 de junho de 2009.

\begin{tabular}{lrr}
\hline Condutas realizadas & N & \% \\
\hline Medicações & 146 & 44,2 \\
Medicações e exames & 61 & 18,4 \\
Exames & 21 & 6,3 \\
Medicações e encaminhamentos & 20 & 6,0 \\
Orientações & 17 & 5,1 \\
Encaminhamentos & 17 & 5,1 \\
Medicações e cuidados & 12 & 3,6 \\
Medicações e orientações & 11 & 3,3 \\
Cuidados & 8 & 2,4 \\
Orientados a retornar no PA & 7 & 2,1 \\
Medicações/ exames/ encaminhamentos & 5 & 1,5 \\
Regulação & 3 & 0,9 \\
Exames e encaminhamentos & 2 & 0,6 \\
\hline Total & $\mathbf{3 3 0}$ & $\mathbf{1 0 0}$ \\
\hline
\end{tabular}

\section{Discussão}

A partir dos dados, observou-se que houve uma pequena predominância dos atendimentos durante $o$ período estudado de usuários do sexo feminino.

Também pôde-se observar uma demanda mais acentuada de usuários entre 20 e 50 anos, representando 63,3\% dos atendimentos. Mas quando foi feito o cruzamento entre os diferentes períodos do dia com a idade dos usuários que procuraram atendimento, foi observado que esta faixa etária procurou o PA nos diferentes períodos do dia, com discreto aumento durante o matutino e vespertino.

O acesso aos serviços de saúde deve ser entendido como a entrada e utilização dos serviços de saúde, e ser levado em um sentido mais amplo, analisando as características da população, o comportamento das pessoas e dos recursos disponíveis. ${ }^{7}$

$\mathrm{O}$ acesso se refere à possibilidade dos usuários utilizarem os serviço de saúde quando precisam. ${ }^{8} \mathrm{Em}$ relação aos dias da semana, pôde-se observar que houve um aumento de atendimentos na segunda-feira. O que de certa forma pode-se pensar na distorção 
do sistema de saúde, já que neste dia as UBS e USF também estão abertas para o atendimento.

E no mesmo sentido, observou-se que de segunda a sexta-feira foram os dias da semana que tiveram maior número de atendimentos no PA, já os finais de semana, quando estas unidades estão fechadas para atendimento, apresentaram um menor número de atendimentos.

Portanto, mesmo que a rede básica feche ao final de semana, este fato não representou aumento expressivo na demanda do PA. Pelo contrário, a segunda-feira aponta a maior demanda, reforçando a necessidade de indagação sobre o motivo da procura pelo PA e de como funciona o vínculo na UBS e USF.

Quanto aos meses de maior procura para atendimento, vale ressaltar que, no ano de 2009, a partir do mês de março iniciou-se a notificação de casos suspeitos ou confirmados de pessoas infectadas pelo vírus da Influenza A, pandemia que foi inédita, divulgada e preocupante nos países latino-americanos. A diferença entre os meses está registrada em março, abril e maio, período da circulação do vírus, havendo modificação considerável entre os meses na frequência de atendimentos no PA durante o período pesquisado.

Foi encontrado que $70,7 \%$ dos atendimentos ocorreram durante o dia, quando a rede básica de atenção está aberta à assistência. Este dado é muito importante para identificar que o usuário na maioria das vezes não procura o PA apenas quando as UBSs e USFs estão fechadas, o que pode ser vislumbrado também no Gráfico 2.

$\mathrm{O}$ relato de dor foi muito frequente, ao mesmo tempo que inespecífico. A dor é considerada uma experiência genuinamente subjetiva e pessoal, e não existe um instrumento padrão que permita a um observador externo, no caso o profissional de saúde, mensurar objetivamente essa experiência interna e complexa. ${ }^{9}$ Portanto, a queixa de dor foi de difícil classificação para avaliação de resultados.

Deste modo, entende-se que as características da dor devem ser bem avaliadas considerando o início, o local, a periodicidade, o tipo de dor, a duração, com o objetivo de os profissionais acolherem de forma mais qualificada as queixas trazidas pelos usuários.

A partir do agrupamento de justificativas realizado neste estudo, se forem somados os motivos da procura classificados como dor, trauma/lesão, problemas respiratórios e problemas gastrointestinais, têmse um total de $62,4 \%$ das justificativas.

Em outras palavras, este percentual representa sintomas como: tosse, resfriado ou gripe, dor de gar- ganta, diarréia, epigastralgia, torção, cefaléias, lombalgia. Portanto, sintomas que representam casos agudos de doença e que não trazem riscos imediatos aos usuários que procuraram atendimento no PA.

Com estes dados, pode-se interpretar que a maioria apresentou queixas de saúde que não necessitariam da alta densidade tecnológica que o PA oferece. ${ }^{10,11}$

Por isso, entende-se que os usuários do PA do distrito oeste procuram a resolução das suas necessidades em saúde em locais onde há maior acesso tanto ao atendimento, quanto à densidade tecnológica, e que há o uso indiscriminado dos recursos que estão disponíveis no serviço de urgência e emergência.

Em relação às condutas, tem-se que em 77,2\% está a terapia medicamentosa associada a outras condutas, sendo que esta, realizada de forma isolada já representa 44,2\%. Logo, o tratamento medicamentoso foi uma constante para os usuários que procuraram o PA. ${ }^{6,12,13}$

A prescrição de medicações, sob a forma de principal conduta, reforça o modelo de atenção baseado na queixa-conduta dos casos agudos, buscando resolver as necessidades de saúde trazidas pelos usuários. Portanto, são condutas de resolução em curto prazo, fato que explica os retornos subsequentes dos mesmos usuários ao PA. Ou seja, quando retornam os sintomas, novamente, os usuários procuram atendimento.

Em contrapartida, 13,2\% das condutas foram de encaminhamentos, seja para outras especialidades, seja para as unidades de atenção primária. Portanto, este é um dado que sugere o PA como um serviço de resolutividade para as diversas queixas de saúde trazidas pelos usuários.

Em 26,3\% das condutas tiveram a solicitação de exames. Logo, esses dados levam a entender que os usuários percebem que no PA conseguem um acesso mais rápido para a realização de seus exames, ao invés de aguardarem ser encaminhados da UBS.

Por outro lado, observou-se que a realização de orientações $(5,1 \%)$ baseadas em tecnologias leves, foi pouco utilizada pelos profissionais em relação aos outros tipos de conduta.

Dessa forma, entende-se que o PA ainda se constitui em um serviço que possui um grande acúmulo de possibilidades de oferta de serviços a serem utilizados pelos usuários, seja na forma de medicamentos, exames e/ou consultas.

Com os dados apresentados, pode-se observar que a terapia medicamentosa fica em evidência dentre as condutas médicas realizadas e que em sua grande 
maioria estão diretamente relacionadas à resolução das queixas relatadas pelos usuários.

Entende-se que, no sentido de compreender as demandas trazidas pelos usuários, ainda há a necessidade de se investir na qualificação dos profissionais do acolhimento que realizam a escuta, a fim de proporcionar um melhor atendimento às necessidades da população em forma de orientações e encaminhamentos.

Em apenas 0,9\% das condutas estão os casos que necessitaram de solicitação de vaga hospitalar pela Central de Regulação de Vagas da cidade de Ribeirão Preto. Este dado aponta uma parcela muito ínfima dos atendimentos que realmente apresentaram gravidade.

Foi observado ainda que em $4,2 \%$ das fichas de atendimento tinha como justificativa para procura pelo PA o retorno para buscar resultado de exames realizados no próprio PA. Portanto, o PA também tem funcionado como um serviço de atendimento como funciona o posto de saúde, onde, após as consultas realizadas, são marcados retornos para os usuários. Esse dado, de certa forma, já aponta a distorção do propósito de um serviço de pronto-atendimento.

Entende-se que a demanda aumentada desses usuários para casos não urgentes no PA, repercute sobre a qualidade da assistência prestada para os casos que realmente necessitam de atendimento de urgência ou emergência. ${ }^{14}$

\section{Conclusões}

Concluí-se que o perfil de usuários atendidos no PA durante o período estudado está bem distribuído em relação ao sexo. Quanto à faixa etária também não houve qualquer aumento expressivo de atendimentos que comprove divergência entre as idades.

Em relação ao dias da semana, observou-se que os dias com maior número de atendimentos estão en- tre segunda à sexta-feira. Quanto ao horário de atendimento comprovou-se que durante todo dia é o período mais procurado, quando as unidades de saúde também estão abertas.

Conclui-se também que os meses entre março e maio apresentam um aumento considerável de atendimentos considerando os casos de suspeita e de confirmados do vírus Influenza A.

Diversas foram as justificativas para a procura pelo PA, no entanto, dentre elas, as potencialidades do PA na forma de alta densidade tecnológica, uma vez que na maioria das condutas está a solicitação de exames e prescrição de medicamentos que são ali mesmo administrados, a fim de atenderam às necessidades de saúde dos usuários que o procuram.

De acordo com o pressuposto de que a demanda aumentada no PA, com casos não urgentes, pode trazer dificuldades para a equipe a fim de proporcionar um atendimento mais acolhedor às necessidades da população, entende-se a importância dos gestores e gerentes de saúde em não economizarem esforços para ampliar o cardápio de ofertas de serviços da atenção básica que atendam aos usuários em suas inúmeras queixas.

Uma estratégia seria promover maiores condições para que após realizarem exames laboratoriais e/ou radiológicos no PA, esses usuários sejam prontamente encaminhados para as UBS e USF para receberem os resultados dos exames e, assim, realizarem o acompanhamento adequado de sua saúde e não somente checarem exames no próprio PA.

E assim, é necessário também investir na qualificação dos profissionais que atuam no PA, a fim de que eles sejam capazes de reorientar os usuários sobre o modelo assistencial atual onde as UBS e USF são a porta de entrada para o sistema em casos que não exijam situações de urgência ou emergência.

\section{ABSTRACT}

The objective of this study was to identify the profile of users treated in emergency care of a district of Ribeirao Preto - SP. Quantitative Study users seeking the emergency care between January to June 2009. Were characterized by sex, age, time, day of week and months of peak demand, demand justification, conduct and referrals. Of the 45,771 visits made, was selected a sample of 330 medical records by random sampling technique. Of these, $57 \%$ of users were female and $63.3 \%$ are users between 20 and 50 years. The months of greatest demand were in March, April and May and $70.7 \%$ of cases occurred during the day. In $20 \%$ of justifications were reports of pain. In $77,2 \%$ of the ducts are drug therapy and $26.3 \%$ test ordering. Among the many justifications for the search by the emergency care are the potential of this service in the form of high-density technology.

Keywords: Primary Health Care. Health Services Accessibility. Emergency Medical Services. 


\section{Referências}

1. Brasil. Ministério da Saúde. O SUS de Aa Z. Garantindo saúde nos municípios. Brasília, MS, 3ํ.ed, 2009.

2. Pinto IC; Rodolpho F; Oliveira MM. Pronto-atendimento: a percepção da equipe de Enfermagem quanto ao seu trabalho no setor de recepção. Rev Gaúch Enferm. 2004; 25: 81-8.

3. Conselho Regional de Medicina. 1988. Disponível em: <http:// www.cremesp. org.br/clippings/legislacao_anteriores_ integra.php>. Acesso em: 09/05/2006.

4. Levin J. Estatística aplicada a ciências humanas. $2^{\circ}$ ed. São Paulo: Harbra, 1987

5. Triola MF. Introdução a estatística. $7^{\circ}$ ed. Rio de Janeiro: LTC, 1999.

6. Ramos DD; Lima MADS. Health care access and receptivity to users in a unit in Porto Alegre, Rio Grande do Sul, Brazil. Cad Saúde Pública. 2003; 19: 27-34.

7. Instituto Nacional de Estadística e Informática. Marco conceptual del acceso a los servicios de salud. In: Determinantes del acceso a los servicios de salud en el peru. [online] Disponível em: http://www.inei.gob.pe/biblioineipub/bancopub/est/ lib0387/indice.htm
8. Gaioso VP, Mishima SM. User satisfaction from the perspective of acceptability in the family health scenario. Texto Contexto - Enferm. 2007; 16:617-25.

9. Sousa FAEF. Pain: the fifth vital sign. Rev Latinoam Enferm. 2002; 10: 446-7.

10. Kovacs MH; Feliciano KVO; Sarinho SW; Veras AACA. Access to basic care for children seen at emergency departments. J. Pediatr. 2005; 81: 251-8.

11. Batistela S; Guerreiro NP; Rossetto NG. Os motivos de procura pelo Pronto Socorro Pediátrico de um hospital Universitário referidos pelos pais ou responsáveis. Semina: Ciências Biológicas e da Saúde. 2008; 29 : 121-30.

12. Tanaka OY, Resenburg CP. Analysis of the use of an ambulatory unit of the Department of Health of the city of São Paulo, Brazil. Rev. Saúde Pública. 1990; 24: 60-8.

13. Souza MF, Pinto IC, Figueiredo LA. Análise da utilização do serviço de pronto-socorro na percepção do usuário. Ciênc Cuid Saúde. 2010; 9:13-20.

14.Puccini, PT. Cornetta, VK. Caseload profile in emergency services: sentinel events for monitoring primary health care. Cad Saúde Pública. [online]. 2008; 24 (9): 2032-42. 